Check for updates

Cite this: Chem. Commun., 2021, 57,367

Received 12th May 2020,

Accepted 4th September 2020

DOI: $10.1039 / \mathrm{d} 0 \mathrm{cc} 03399 \mathrm{e}$

rsc.li/chemcomm

\section{Anti-drug antibody detection with label-free electrolyte-gated organic field-effect transistors $\dagger$}

\author{
Matteo Sensi, (D) a Marcello Berto, (D) a Sara Gentile, ${ }^{a}$ Marcello Pinti, ${ }^{a}$ \\ Andrea Conti, DD ${ }^{\mathrm{b}}$ Giovanni Pellacani, ${ }^{\mathrm{b}}$ Carlo Salvarani, ${ }^{\mathrm{C}}$ Andrea Cossarizza, ${ }^{\mathrm{d}}$ \\ Carlo Augusto Bortolotti (D) ${ }^{a}$ and Fabio Biscarini (D) *ae
}

The efficacy of immunotherapy can be undermined by the development of an immune response against a drug/antibody mediated by anti-drug antibodies (ADAs) in treated patients. We present the first label-free EGOFET immunosensor that integrates a biological drug, Nivolumab (Opdivo $($ )), as a specific recognition moiety to quantitatively and selectively detect ADAs against the drug. The limit of detection is $100 \mathrm{fM}$. This demonstration is a prelude to the detection of ADAs in a clinical setting in the treatment of different pathologies, and it also enables rapid screening of biological drugs for immunogenicity.

The recent development of biotechnological drugs is greatly improving the pharmacological treatment of many diseases. An example of this therapeutic innovation is immunotherapy, which has been proven effective against some types of cancer (melanoma, lung, colon, liver, kidney, and head) ${ }^{1-3}$ and inflammatory chronic diseases (psoriasis, rheumatoid arthritis, Crohn's disease, and systemic lupus erythematosus). ${ }^{4-6}$

In tumors, cancer cells can evade the immune surveillance thanks to different resistance mechanisms, including the stimulation of immune checkpoints. Immune checkpoints are essential for self-tolerance, which prevents the indiscriminate action of the immune system towards any cell in an organism and is central in autoimmune diseases. It has been demonstrated that inhibiting immune checkpoints induces a large $\mathrm{T}$ cell response against cancer cells. ${ }^{7}$ In 2011, the $\mathrm{FDA}^{8}$ approved

\footnotetext{
${ }^{a}$ Dipartimento di Scienze della Vita - Università di Modena e Reggio Emilia, Via Campi 103, 41125 Modena, Italy.E-mail: fabio.biscarini@unimore.it

${ }^{b}$ Dipartimento Medicine Specialistiche, S.C. Dermatologia, Azienda Ospedaliero Universitaria di Modena, Via Del Pozzo 71, 41121 Modena, Italy

${ }^{c}$ Unità Reumatologica, Università di Modena e Reggio Emilia, Azienda USL-IRCCS di Reggio Emilia, Modena, Italy

${ }^{d}$ Dipartimento di Scienze Mediche e Chirurgiche Materno-Infantili e dell'Adulto, Università di Modena e Reggio Emilia, Via Campi 287, 41125 Modena, Italy

${ }^{e}$ Center for Translational Neurophysiology, Istituto Italiano di Tecnologia, Via Fossato di Mortara 17-19, 44100 Ferrara, Italy

† Electronic supplementary information (ESI) available. See DOI: 10.1039/ docc03399e
}

the first clinically validated checkpoint inhibitor called Ipilinumab, a monoclonal antibody that blocks the receptor Cytotoxic T-lymphocyte-associated antigen 4 (CTLA-4), an immune checkpoint that downregulates the immune response. Another approved checkpoint inhibitor is Nivolumab (Opdivo $\mathbb{C}$ ), ${ }^{9}$ which shows promising results in the treatment of melanoma, Hodgkin's lymphoma, non-small cell lung cancer, ${ }^{1,10-12}$ renal cell carcinoma, liver cancer, bladder cancer, head and neck cancer and colon cancer. Nivolumab is a fully human IgG4 monoclonal antibody which blocks the Programmed cell-death protein 1 (PD-1), an inhibitory transmembrane protein expressed by $\mathrm{T}$ cells. Binding of PD-1 to its cognate ligands PD-L1/2, ${ }^{13}$ highly expressed by cancer cells, triggers several mechanisms that dampen the immune response, such as exhaustion of peripheral $\mathrm{T}$ effector cells and conversion of effector $\mathrm{T}$ cells to regulatory $\mathrm{T}$ cells. ${ }^{14,15}$ Thus, blockade of the PD-1 pathway by Nivolumab enhances the $T$ cell anti-tumor activity and thereby favours killing of cancer cells. ${ }^{16,17}$ Unfortunately, a side effect of Nivolumab and other checkpoint inhibitors is the development of an immune response against the drug itself, mediated by anti-drug antibodies (ADAs). ${ }^{18-20}$ ADAs are found in $12.7 \%$ of patients under Nivolumab therapy ${ }^{19}$ and their detection is not trivial mainly due to the absence of a homogeneous ADA population and concentration (ranging from few $\mathrm{ng} \mathrm{ml}^{-1}$ to almost $1 \mu \mathrm{g} \mathrm{ml} \mathrm{ml}^{-1}$ ) and the technical difficulty of detecting ADAs once in complex with the drug. The main strategies currently used to detect ADAs are ELISA, homogeneous mobility shift assay (HMSA), electrochemiluminescence (ECL) immunoassay, and cell-based reporter-gene assay (RGA). ${ }^{18,21}$ All these methods allow the detection of ADAs with a minimum LOD between $10 \mathrm{pM}$ and $100 \mathrm{pM}$, but they require labels (fluorescent dyes and enzymes), specialized laboratories and/or qualified personnel. The development of a fast biosensor to detect ADAs in the patient plasma with a point of care (POC) approach could facilitate the early detection of adverse immune responses. In the field of POC biosensors, organic electronics is an attractive platform because it enables the fabrication of devices that are ultrasensitive and biocompatible, 
and that can be made flexible and impalpable, and fabricated in a low cost, high throughput method. Electrolyte-gated organic field-effect transistors (EGOFETs) are among the most widely explored device architectures for biosensing with organic electronics. ${ }^{22-24}$ EGOFETs are three-electrode devices operated in a capacitive coupling. An organic semiconductor channel transduces a small voltage bias into a large signal in the presence of an electrolyte. Functionalization of a gate electrode with a recognition moiety, such as an antibody, an aptamer, an enzyme, or a whole virus or bacterium, can make its response selective towards a given target, a necessary requisite for its operation as a biosensor. Thanks to their ability to amplify small signals and the low limit of detection, EGOFETs were shown to be state-of-the-art sensors for the detection of organic chemicals, ${ }^{25}$ metals, ${ }^{26}$ water pollutants, ${ }^{27}$ proteins, ${ }^{23,28,29}$ nucleic acids ${ }^{30}$ and viruses. ${ }^{31}$

In this work, we show the first EGOFET immunosensor able to detect ADAs; in particular, we validate the biosensor for the detection of commercial anti-Nivolumab antibodies. The device embodies an Au gate functionalized to immobilize Nivolumab as an ADA specific probe. By measuring the electrical characteristics of the device exposed to ADAs in buffer, we obtain a dose curve enabling anti-Nivolumab ADAs across four orders of magnitude with a theoretical limit of detection as low as $100 \mathrm{fM}$. This work indicates a possibility to dose ADAs with a sensing platform that is portable and easy to upscale, can be tailored to clinicians' needs, and can be used at the POC without any particular training of the personnel.

EGOFETs were patterned on a quartz substrate, featuring two interdigitated (width-to-length ratio $W / L=500$ ) gold electrodes as the source and the drain. The semiconductor channel was made of TIPS-pentacene thin films by spin-coating. To perform specific biosensing, we functionalized the gate electrode with the drug Nivolumab as the probe. As a first step, we chemisorbed Cys-protein $\mathrm{G}$ on the gold surface of the gate by means of the thiol group of an engineered exposed cysteine that binds to the $\mathrm{Au}$ electrode. Since protein $\mathrm{G}$ binds specifically to the Fc region of antibodies, the binding geometry of Nivolumab results in exposure to the solution of the Fab region, which is the docking site of ADAs. In the second step, the free gold surface was saturated with an OEG (11-mercaptoundecyltriethylene glycol) self-assembled monolayer (SAM) (see Fig. 1), with the aim to minimize nonspecific binding of Nivolumab and ADAs on the surface of the gate electrode. This step is necessary to minimize, if not deplete completely, the non-specific binding of the ADAs on gold (see Fig. S2, ESI $\dagger$ ). Cyclic voltammetry and electrochemical impedance spectroscopy measurements in the presence of the redox probe $\mathrm{K}_{3}\left[\mathrm{Fe}(\mathrm{CN})_{6}\right]$ were used to assess the functionalization strategy by monitoring the increased resistance to charge transfer following each functionalization step. As shown in Fig. 2a, chemisorption of protein $\mathrm{G}$ decreases the redox peak intensity, and the partial surface coverage ( $\sim 40 \%$ ) can be inferred. Since the formation of the OEG monolayer leads to almost full passivation of the electrode, binding of Nivolumab to protein $G$ only induces minor further changes.

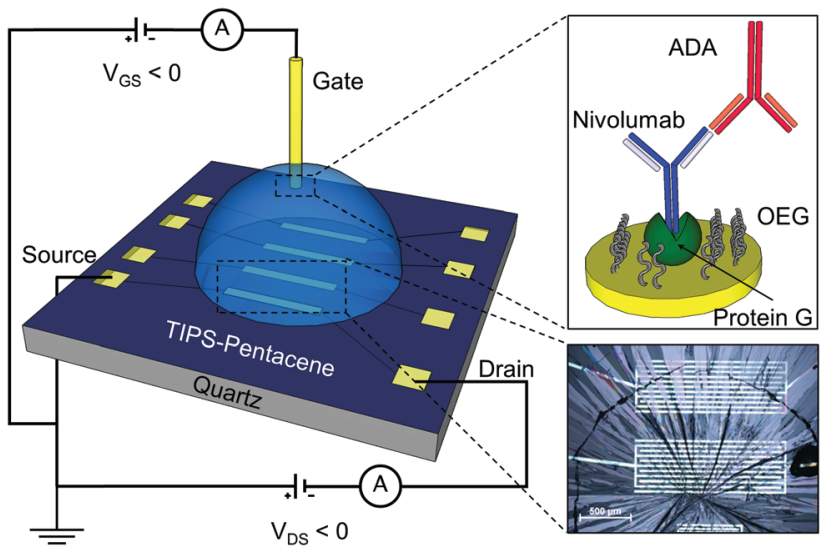

Fig. 1 EGOFET and the gate functionalization scheme. The EGOFET was fabricated on a quartz substrate, with interdigitated drain and source gold electrodes. A TIPS-pentacene OSC thin film covers the channel (optical microscopy picture in the inset). A PBS drop connects the channel and the top-gate electrode. The gate, as shown in the top inset, is functionalized with protein $G$ and OEG monolayers, the Fc region of Nivolumab binds the protein $G$, ensuring an effective orientation (see procedure steps in Fig. S1, ESI $\dagger$ )

The biosensing activity was tested by incubating the functionalized gate in solutions of ADAs at different concentrations, from $1 \mathrm{pM}$ to $10 \mathrm{nM}$, for 20 minutes each. This time is normally enough for validating the device and the procedure does not affect its performance (Fig. S5, ESI $\dagger$ ). We used anti-Nivolumab ADAs that are recombinant, inhibitory anti-idiotypic monoclonal antibodies in the monovalent Fab format $(\sim 50 \mathrm{kDa})$. This form was chosen to avoid their binding to proteins $G$ and so test the specificity of the probe. By recording the transfer characteristics after each incubation, we observed a decrease in the drain-source current $\left(I_{\mathrm{DS}}\right)$ and a shift of the threshold voltage $\left(V_{\mathrm{TH}}\right)$ (Fig. 3, panel a). The response of the biosensor is expressed as $S=-\Delta I / I_{0}$, where $I_{0}$ is the $I_{\mathrm{DS}}$ current measured at $[\mathrm{ADA}]=0$, while $-\Delta I$ is the difference between $I_{\mathrm{DS}}$ at a specific ADA concentration and $I_{0}$, and the gate bias is $V_{\mathrm{GS}}=-0.2 \mathrm{~V}$ and $V_{\mathrm{DS}}=-0.2 \mathrm{~V}$ (linear regime). The response reveals a Hill-type behaviour, with saturation at higher concentrations (see Fig. 3, panel b). By fitting the Hill equation to the data:

$$
S=S_{\max } \frac{\left(K_{\mathrm{H}}^{\alpha}[\mathrm{ADA}]^{\alpha}\right)}{1+K_{\mathrm{H}}^{\alpha}[\mathrm{ADA}]^{\alpha}}
$$

we obtained a binding association constant $K_{\mathrm{H}}$ value of $(5.1 \pm 0.9) \times$ $10^{11} \mathrm{M}^{-1}$, with a cooperativity exponent $\alpha=0.64 \pm 0.07$. By fitting the Langmuir equation to the data (Fig. 3b):

$$
S=S_{\max } \frac{K_{\mathrm{L}}[\mathrm{ADA}]}{1+K_{\mathrm{L}}[\mathrm{ADA}]}
$$

we obtained a binding association constant $K_{\mathrm{L}}$ of $(4.5 \pm 1.0) \times$ $10^{11} \mathrm{M}^{-1}$, comparable to the value obtained by fitting the data with the Hill-type model.

The available commercial ELISA kit (for research purpose only) helps in qualitative (Abcam, Biovision, ELISA Genie) or semi-quantitative (IBL-America, sensitivity $\sim 67 \mathrm{pM}$ ) detection of anti-Nivolumab ADAs in the serum or plasma. Our device 
a)

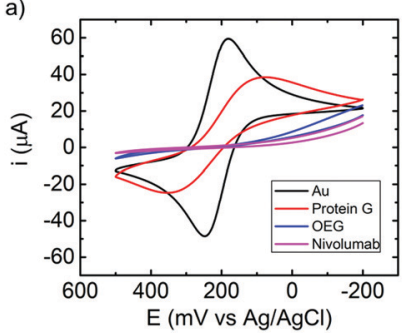

b)

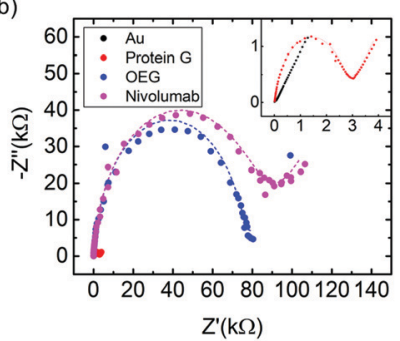

Fig. 2 Monitoring of functionalization steps by electrochemical methods (a) Cyclic voltammetry and (b) electrochemical impedance spectroscopy of the bare gate electrode (black) and after successive functionalization by incubation with protein $\mathrm{G}\left(2 \mathrm{~h}, 2.5 \mathrm{mg} \mathrm{m}^{-1}\right.$, red curve), OEG (20 $\mathrm{min}$, $100 \mu \mathrm{M}$, blue) and Nivolumab ( $2 \mathrm{~h}, 1 \mathrm{mg} \mathrm{ml}^{-1}$, magenta). The dashed lines in panel (b) denote the fit obtained with the Randles circuit.
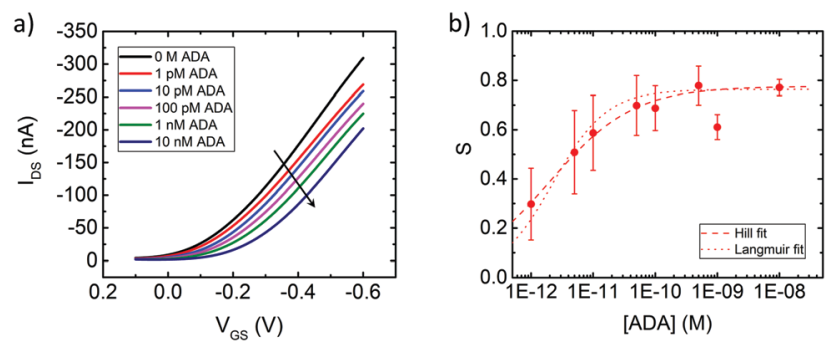

Fig. 3 Transfer characteristics and response of the device to ADA. (a) Transfer curves of the device after incubation at different concentrations of anti-Nivolumab ADAs, at $V_{D S}=-0.2 \mathrm{~V}$. (b) Semilog plot of the signal $S$ vs. [ADAs] molar concentration at $V_{G S}=-0.2 \mathrm{~V}$. The dashed curve denotes the best fit with the Hill equation. Each data point represents the average of 4 different devices, at each concentration (only exceptions are 1 pM, 10 pM and 100 pM with 8 devices), while the error bars are the standard deviation.

can detect ADAs in PBS in $\mathrm{pM}$ range concentrations, with a limit of detection (LOD) $=100 \mathrm{fM}$, which is well within the physiopathological range.

We determined the sensitivity of the device as a numerical derivative $\mathrm{d} S / \mathrm{d}[\mathrm{ADA}]$ (see Fig. S3, ESI $\dagger$ ). The sensitivity is higher at lower concentrations of ADA $\left(1 \times 10^{11} \mathrm{M}^{-1}\right.$ at $\left.1 \mathrm{pM} \mathrm{ADA}\right)$, while it decreases by 4 orders of magnitude in the nanomolar range.

By applying a linear fit on the transfer curves, we extracted the transconductance $\left(g_{\mathrm{m}}\right)$ from the slope and the threshold voltage $\left(V_{\mathrm{TH}}\right)$ from the intercept between the baseline current (off-current) and the transfer curve, upon incubation of the gate with each ADA solution (Fig. S8a, ESI $\dagger$ ). The transconductance as a function of ADA concentration presents a small decrease $(-0.05 \mu \mathrm{S})$ at first exposure to ADAs $(1 \mathrm{pM})$ and then saturates to a plateau. On the contrary, the threshold voltage shifts at larger absolute values, following a power law trend as a function of ADA concentration. This behaviour of the transistor parameters was confirmed independently by extracting them from the maximum of the first derivative (for $g_{\mathrm{m}}$ ) and the minimum of the second derivative (for $V_{\mathrm{TH}}$ ) of the transfer curve (see Fig. S4, ESI $\dagger$ ).

The absence of effects on the transconductance of the device and the negative shift of the threshold voltage from about $0 \mathrm{~V}$ to

$-0.25 \mathrm{~V}$ over four decades of ADA concentration, both suggest that the binding of ADAs to the drug immobilized on the gate shifts its electrochemical potential downward, leading to lesser mobile holes in the p-type semiconductor. ${ }^{29}$ This may be rationalized by assuming that a negative charge results from the ADA/Nivolumab adduct, thus perturbing the potential within the electrical double layer at the interface with the gate electrode, e.g. screening or enhancing the field created by the gate potential. The result is that an opposite change (in this case positive) is generated in the electrical double layer at the interface between the organic semiconductor and the electrolyte. This effectively corresponds to the increase of the Fermi level in the OSC. From a microscopic point of view, the extra positive charge will increase the energy of the polaron levels (p-type) and/or lower the energy of the neutral states, thus enlarging the hopping barrier. The outcome is that the current will exponentially decrease, which indeed agrees with the experimental observation.

Hence, the $V_{\mathrm{TH}}$ shift appears to be a suitable parameter for monitoring the ADA concentration, as it scales linearly with the signal, and exhibits no saturation in the $\mathrm{nM}$ range of the ADA concentration. Fig. S8b (ESI $\dagger$ ) shows a comparison of the $V_{\mathrm{TH}}$ extracted either from the linear fit of $I_{\mathrm{DS}}$ (squares) or $I_{\mathrm{DS}}{ }^{1 / 2}$ (circles). Both exhibit a linear correlation with the signal $S$ with a similar slope (sensitivity) for the same gate voltage used. By changing the method to extract the $V_{\mathrm{TH}}$, we observe a shift of $200 \mathrm{mV}$, while the sensitivity and the signal trend are conserved. The steeper lines, exhibiting higher sensitivity, are the ones corresponding to the sub-threshold regime $\left(V_{\mathrm{GS}}=-0.1 \mathrm{~V}\right)$.

To determine the selectivity of the Nivolumab-ADA binding, we tested the response of the device to another anti-drug antibody, anti-infliximab, also featuring the Fc fragment. As shown in Fig. 4a, incubation of the gate in anti-infliximab ADA (ATIs, black) solutions leads to a current decrease that is much lower, hence clearly distinguishable, than the signal due to the recognition of specific anti-Nivolumab ADAs (red). The mostly linear signal observed for anti-infliximab ADAs is probably related to non-specific interactions with Nivolumab, protein G or the electrode surface. The absence of specific interaction
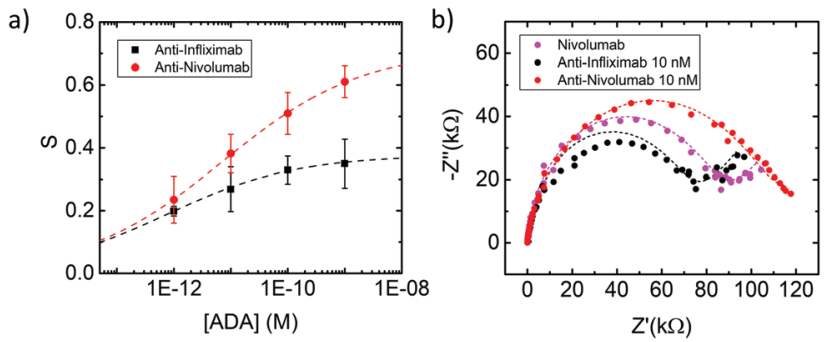

Fig. 4 Selectivity of detection. Comparison between the response of the device towards anti-Nivolumab (red) and anti-infliximab (black) ADAs based on (a) the signal calculated from the transfer curves (see Fig. S6a, $E S I \dagger$ ), in the semilog plot at $V_{G S}=-0.1 \mathrm{~V}$ and $V_{D S}=-0.2 \mathrm{~V}$ and (b) faradaic electrochemical impedance spectroscopy. The error bars denote the standard deviation on the average of three devices. In both panels the $\mathrm{Au}$ electrodes were functionalized following the procedure shown in Fig. $\mathrm{S} 1(\mathrm{ESI} \dagger)$. 
between ATIs and the device was confirmed by electrochemical impedance spectroscopy (Fig. 4b), by exposing the gate functionalized with protein G, Nivolumab and OEG to $10 \mathrm{nM}$ antiinfliximab antibodies for 20 minutes. After recording the EIS spectrum, the same gate was exposed for $20 \mathrm{~min}$ to $10 \mathrm{nM}$ antiNivolumab antibodies. The binding of anti-infliximab to the Nivolumab-functionalized gate has a small effect on the charge transfer resistance, the imaginary component of impedance is not affected ( $Z_{\mathrm{im}}$ in Fig. S6b, ESI $\dagger$ ), while the real component impedance $\left(Z_{\text {re }}\right.$ in Fig. S6b, ESI $\dagger$ ) decreases at $1 \mathrm{~Hz}$ by $10 \mathrm{k} \Omega$. The binding of anti-Nivolumab to the Nivolumab-functionalized gate increases both the imaginary component of impedance at $1 \mathrm{~Hz}$ by $20 \mathrm{k} \Omega$ and the real component impedance by $10 \mathrm{k} \Omega$, supporting the evidence of the decrease of capacitance and the increase of charge transfer resistance. We also observed a negligible signal by performing a sensing experiment, as a control, with a device where the gate was functionalized with infliximab and exposed to anti-Nivolumab ADAs (see Fig. S7, ESI $\dagger$ ).

In summary, this work shows that it is possible to dose ADAs for a specific biological drug/antibody with high accuracy (LOD $=100 \mathrm{fM})$, sensitivity $\left(1 \times 10^{11} \mathrm{M}^{-1}\right.$ at $1 \mathrm{pM}$ ADA $)$ and selectivity. This outstanding sensing performance is enabled by the characteristic properties of organic semiconductors; in particular, the tail of the density of states (DOS) of the semiconductor imparts exponential amplification to minute changes of the potential at the interface between the semiconductor and the electrolyte. ${ }^{28}$ These potential changes originate in the electrolyte gated organic transistor by the displacement of ions at the interface between the organic semiconductor and the electrolyte, in response to the changes of the electrochemical potential at the gate electrode caused by biorecognition events. The ultrasensitivity and ease of operation make the organic sensors particularly apt for the POC testing or for the in-field deployed application, a scenario that is very common nowadays. The capability of detecting antibodies against a biological drug makes the technology attractive for planning, monitoring, and re-directing therapeutic plans based on biological drugs. It can also serve for experimental screening of candidate biological drugs with an in vitro assay that allows ranking them by their immunogenicity. The impact of such sensing technology is in principle enormous, as it can find applications in urgent scenarios such as the COVID-SARS2 virus infection (COVID-19 disease). The next steps will be clinical validation with patients' samples and benchmarking against state-of-the-art assays for ADA quantification.

\section{Conflicts of interest}

There are no conflicts to declare.

\section{References}

1 K. M. Mahoney, G. J. Freeman and D. F. McDermott, Clin. Ther., 2015, 37, 764-782.

2 P. J. Medina and V. R. Adams, Pharmacotherapy, 2016, 36, 317-334.

3 A. Karlsson and S. Saleh, Clin. Cosmet. Investig. Dermatol., 2017, 10, 325-339.

4 T. T. Atsushi Ogata, Rheumatol. Curr. Res., 2014, S4.

5 S. Candon, A. Mosca, F. Ruemmele, O. Goulet, L. Chatenoud and J. P. Cézard, Clin. Immunol., 2006, 118, 11-19.

6 L. S. Winterfield and A. Menter, Dermatol. Ther., 2004, 17, 409-426.

7 D. M. Pardoll, Nat. Rev. Cancer, 2012, 12, 252-264.

8 F. Cameron, G. Whiteside and C. Perry, Drugs, 2011, 71, 1093-1104.

9 C. Wang, K. B. Thudium, M. Han, X.-T. Wang, H. Huang, D. Feingersh, C. Garcia, Y. Wu, M. Kuhne, M. Srinivasan, S. Singh, S. Wong, N. Garner, H. Leblanc, R. T. Bunch, D. Blanset, M. J. Selby and A. J. Korman, Cancer Immunol. Res., 2014, 2, 846-856.

10 L. J. Scott, Drugs, 2015, 75, 1413-1424.

11 P. Darvin, S. M. Toor, V. Sasidharan Nair and E. Elkord, Exp. Mol. Med., 2018, 50, 1-11.

12 A. Hauschild and C. Garbe, Nat. Rev. Clin. Oncol., 2015, 12, 439-440.

13 S. Gandini, D. Massi and M. Mandalà, Crit. Rev. Oncol. Hematol., 2016, 100, 88-98.

14 L. M. Francisco, V. H. Salinas, K. E. Brown, V. K. Vanguri, G. J. Freeman, V. K. Kuchroo and A. H. Sharpe, J. Exp. Med., 2009, 206, 3015-3029.

15 S. Amarnath, C. W. Mangus, J. C. M. Wang, F. Wei, A. He, V. Kapoor, J. E. Foley, P. R. Massey, T. C. Felizardo, J. L. Riley, B. L. Levine, C. H. June, J. A. Medin and D. H. Fowler, Sci. Transl. Med., 2011, 3, 1-14.

16 L. Guo, H. Zhang and B. Chen, J. Cancer, 2017, 8, 410-416.

17 M. A. Postow, M. K. Callahan and J. D. Wolchok, J. Clin. Oncol., 2015, 33, 1974-1982.

18 E. M. J. van Brummelen, W. Ros, G. Wolbink, J. H. Beijnen and J. H. M. Schellens, Oncologist, 2016, 21, 1260-1268.

19 S. Agrawal, P. Statkevich, G. Bajaj, Y. Feng, S. Saeger, D. D. Desai, J. S. Park, I. M. Waxman, A. Roy and M. Gupta, J. Clin. Pharmacol., 2017, 57, 394-400.

20 M. Tocut, R. Brenner and G. Zandman-Goddard, Autoimmun. Rev., 2018, 17, 610-616.

21 B. Gorovits, D. J. Baltrukonis, I. Bhattacharya, M. A. Birchler, D. Finco, D. Sikkema, M. S. Vincent, S. Lula, L. Marshall and T. P. Hickling, Clin. Exp. Immunol., 2018, 192, 348-365.

22 M. Berto, C. Diacci, R. D’Agata, M. Pinti, E. Bianchini, M. Di Lauro, S. Casalini, A. Cossarizza, M. Berggren, D. Simon, G. Spoto, F. Biscarini and C. A. Bortolotti, Adv. Biosyst., 2017, 1700072.

23 S. K. Sailapu, E. Macchia, I. Merino-Jimenez, J. P. Esquivel, L. Sarcina, G. Scamarcio, S. D. Minteer, L. Torsi and N. Sabaté, Biosens. Bioelectron., 2020, 156, 1-7.

24 D. Wang, V. Noël and B. Piro, Electronics, 2016, 5, 9.

25 S. Casalini, F. Leonardi, T. Cramer and F. Biscarini, Org. Electron. physics, Mater. Appl., 2013, 14, 156-163.

26 T. T. K. Nguyen, H. V. Tran, T. T. Vu, S. Reisberg, V. Noël, G. Mattana, M. C. Pham and B. Piro, Biosens. Bioelectron., 2019, 127, 118-125.

27 T. T. K. Nguyen, T. N. Nguyen, G. Anquetin, S. Reisberg, V. Noël, G. Mattana, J. Touzeau, F. Barbault, M. C. Pham and B. Piro, Biosens. Bioelectron., 2018, 113, 32-38.

28 M. Berto, S. Casalini, M. Di Lauro, S. L. Marasso, M. Cocuzza, D. Perrone, M. Pinti, A. Cossarizza, C. F. Pirri, D. T. Simon, M. Berggren, F. Zerbetto, C. A. Bortolotti and F. Biscarini, Anal. Chem., 2016, 88, 12330-12338.

29 E. Macchia, K. Manoli, B. Holzer, C. Di Franco, M. Ghittorelli, F. Torricelli, D. Alberga, G. F. Mangiatordi, G. Palazzo, G. Scamarcio and L. Torsi, Nat. Commun., 2018, 9, 3223.

30 L. Kergoat, B. Piro, M. Berggren, M. Pham and A. Yassar, Org. Electron., 2012, 13, 1-6.

31 M. Berto, E. Vecchi, L. Baiamonte, C. Condò, M. Sensi, M. Di Lauro, M. Sola, A. De Stradis, F. Biscarini, A. Minafra and C. A. Bortolotti, Sens. Actuators, B, 2019, 281, 150-156. 